\title{
EDUCAÇÃO EM SAÚDE PARA CRIANÇAS COM ESTOMIAS INTESTINAIS: O ENFERMEIRO COMO MEDIADOR DO CUIDAR
}

\author{
HEALTH EDUCATION FOR CHILDREN WITH INTESTINAL STOMIES: \\ THE NURSE AS CAREGIVER OF CARE
}

\author{
Sandra de Nazaré Costa Monteiro ${ }^{1}$ \\ Elisabete Mesquita Peres Carvalho ${ }^{2}$ \\ Laiane Medeiros ${ }^{3}$ \\ Ana Lúcia da Silva ${ }^{4}$ \\ Dirce Guilhem ${ }^{5}$
}

\begin{abstract}
Resumo: Este estudo analisou a produção científica nacional e internacional desenvolvidos pela enfermagem acerca da educação em saúde para crianças com estomias intestinais. Trata-se de uma revisão integrativa de publicações nas bases do portal da Biblioteca Virtual de Saúde, PubMed, Literatura LatinoAmericana e do Caribe em Ciências da Saúde, Medical Literature Analysis and Retrieval System Online e Cinahl, em português, inglês e espanhol, de janeiro de 2013 a dezembro de 2016. A busca resultou em 125 artigos dos quais 9 cumpriram os critérios de inclusão. Da análise surgiram quatro categorias: (1) educação em saúde; (2) atuação do enfermeiro; (3) Plano de cuidados; e (4) Inserção social. A educação em saúde para pais/cuidadores é primordial para o desenvolvimento saudável de crianças com estomias.
\end{abstract}

Palavras-chave: Criança; Estomia; Educação em saúde; Enfermagem; Revisão Integrativa.

Abstract: This study analyzed the national and international scientific production developed by nursing on health education for children with intestinal stomies. This is an integrative review of publications in the databases of the Virtual Health Library, PubMed, Latin American and Caribbean Literature in Health Sciences, Medical Literature Analysis and Retrieval System Online and Cinahl in Portuguese, English and Spanish, from January 2013 to December 2016. The search resulted in 125 articles, of which 9 fulfilled the inclusion criteria. Four categories emerged from the analysis: (1) health education; (2) nurses' performance; (3) Care plan; and (4) Social insertion. Health education for parents / caregivers is paramount for the healthy development of children with stomies.

Keywords: Child; Stomach; Health education; Nursing; Integrative Revision.

\footnotetext{
${ }^{1}$ Doutoranda do Programa de Pós-graduação DINTER em Ciências da Saúde da Universidade de Brasília (UnB), Brasília, Distrito Federal, Brasil. E-mail: sncmonteiro@yahoo.com.br

${ }^{2}$ Doutoranda do Programa de Pós-graduação DINTER em Ciências da Saúde da Universidade de Brasília (UnB), Basília, Distrito Federal, Brasil. E-mail: elisabete_mpc@ yahoo.com.br

${ }^{3}$ Doutora pela USP Ribeirão Preto. Docente do Curso de Enfermagem da Universidade de Brasília (UnB), Brasília, Distrito Federal, Brasil. E-mail: lainha@gmail.com

${ }^{4}$ Doutora em Ciências Médicas UnB. Docente do Curso de Enfermagem da Universidade de Brasília (UnB), Brasília, Distrito Federal, Brasil. E-mail: analucia@unb.br

${ }^{5}$ Pós-Doutora em Bioética pela Uniservidad Complutense de Madrid. Docente do Curso de Enfermagem da Universidade de Brasília (UnB), Brasília, Distrito Federal, Brasil. E-mail: dirceguilhem@gmail.com
} 


\section{Introdução}

A confecção de uma estomia é a exteriorização de um segmento de qualquer víscera oca do corpo, e de acordo com a sua procedência recebe nomes diferenciados, para estomias intestinais têm-se a colostomia, ileostomia e a jejunostomia. Já as estomias urinárias denominam-se urostomia ou derivação urinária. É uma situação que pode acometer diferentes faixas etárias, e uma grande variedade de situações clínicas, na criança a maioria das vezes é utilizada para tratamento de doenças benignas e de caráter temporário (SANTOS; CESARETTI, 2015).

A inexistência de um cadastro nacional único que forneça dados epidemiológicos acerca de estomias pediátricas, dificulta a visibilidade desse quantitativo, além de dificultar propostas assistenciais, educacionais e políticas para essa clientela. Sabe-se, porém, que em países desenvolvidos, crianças com anomalias congênitas que necessitam da confecção de estomia, tem maior incidência no sexo masculino quando comparado ao feminino $(6,1 \%$ e $4,0 \%$ respectivamente); e maior incidência em negras comparativamente às brancas $(6,4 \%$ e $4,9 \%$ respectivamente), além de cerca de $25 \%$ das admissões em hospitais pediátricos serem de crianças com malformações congênitas (CMR, 2006).

Em estudo recente no norte do Brasil acerca do perfil sociodemográfico e clínico dessas crianças constatou-se que a maioria era do sexo masculino, na faixa-etaria de 0 a 3 anos, sem escolaridade, tendo como causa básica para a cirugia geradora da estomia a malformação congênita, resultando em colostomia terminal de duas bocas em carácter temporário (BEZERRA et al., 2017). No Distrito Federal-Brasil, pesquisa semelhante realizada em um ambulatório de estomaterapia de um hospital público, em 2013, fundamentado em 50 prontuários de crianças e adolescentes com estomia, revelou que $62 \%$ era do sexo masculino. A maioria estava na faixa etária de 0 a 5 anos (84\%), e $60 \%$ era oriundo de áreas administrativas do DF (MONTEIRO et al., 2014).

Destaca-se também que $90 \%$ de crianças estomizadas atingem a vida adulta. Assim, os cuidadores/familiares necessitam estar aptos para esses cuidados específicos no ambiente domiciliar, onde uma estratégia utilizada é a criação de parceria entre os profissionais de saúde, a fim de amenizar os desgastes do cuidado a uma criança estomizada ainda no ambiente hospitalar (FONSECA; MARCON, 2009).

O paciente pediátrico possui caraterísticas próprias por se tratar de um ser em evolução e contínuo processo de adaptação da estrutura à função. Se modifica dos adultos 
por diferenças anatômicas, psicológicas, fisiológicas e elementos patológicos. Uma intervenção cirúrgica representa uma situação crítica, sendo que na população infantil isso é pior por ela ser mais sensível, possuir desenvolvimento imaturo e recursos limitados para o enfrentamento de situações desconhecidas e dolorosas (KAYO et al., 2015).

Pelo caráter temporário, a estomia pediátrica torna-se um desafio para a criança, a família e para os profissionais de saúde, que precisam conviver por um tempo de permanência indeterminado nessa condição. Ao mesmo tempo, segue o processo normal de crescimento e desenvolvimento saudável da criança concomitante a necessidade de capacitação técnica que a equipe deve oferecer aos pais para que continuem o cuidado com a estomia no domicílio.

Nesse sentido, torna-se necessário proporcionar recursos que facilitem a percepção da realidade e de seu propósito, bem como dar-lhe apoio que lhe permitam expressar em segurança e de acordo com o nível de desenvolvimento, as emoções decorrentes dela. Quando a criança compreende a verdadeira finalidade do procedimento, é capaz de tolerar melhor o desconforto e a dor.

A avaliação da criança estomizada é realizada por uma equipe multiprofissional, e nesse contexto o enfermeiro possui a competência e a habilidade para avaliar as condições do estomizado, por meio do exame físico e de uma avaliação detalhada das informações a respeito do cuidado com estomias, hábitos alimentares, adaptação a nova condição de vida, apoio emocional e convivência social. Identifica também as necessidades da criança e da família, na qual é possível descortinar um novo olhar para outras estratégias terapêuticas em benefício do bem-estar e da qualidade de vida daquela família (MATSUBARA et al., 2012).

Para mediar o trabalho do enfermeiro na educação em saúde, utilizam-se tecnologias educacionais, que servirão como dispositivos de mediação para os facilitadores na disseminação da informação e formação, e, sua utilização, aplicação envolve atitudes que possibilitem atender o público alvo a quem se destina (TEIXEIRA; MEDEIROS; NASCIMENTO, 2014).

Nesse sentido, as tecnologias assumem um papel importante no processo de educar em saúde, pois facilitam a mediação de conteúdos de aprendizagem, funcionam como recurso prontamente disponível para que a criança e seu familiar possam consultálos, diante de dúvidas no desenvolvimento da prestação do cuidado. Sendo o assim, a pesquisa teve como objetivo analisar, na produção científica nacional e internacional, 
estudos desenvolvidos pela enfermagem acerca da educação em saúde para crianças estomizadas.

Diante do contexto elaborou-se como questão de pesquisa: o que há na literatura acerca de educação em saúde para criança estomizada e seus familiares nos últimos quatro anos?

\section{Método}

Trata-se de uma revisão integrativa de literatura que vem contribuir com o aprofundamento do conhecimento sobre as produçoes científicas relativas a uma determinada temática e consiste na contrução de uma análise ampla da literatura, para as discussões sobre conceitos, revisão de teorias, métodos e resultados de pesquisas, assim com reflexes sobre a realização de futuros estudos, o que contribui para a análise dos estudos realizados área da enfermagem (MENDES; SILVEIRA; GALVÃO, 2008).

Inicialmente, definiu-se a questão de pesquisa: $\mathrm{O}$ que evidenciam as produções científicas nacionais e internacionais acerca de educação em saúde para criança estomizada e seus familiares nos últimos quanto anos?

Em seguida, foram estabelecidas as bases de dados, utilizando-se como critério estas publicarem artigos de relevância na área da saúde. A busca bibliográfica foi realizada nos portais de Scopus, Biblioteca Virtual de Saúde (BVS) e PubMed, na qual foram consultadas as bases de dados da Literatura Latino-Americana e do Caribe em Ciências da Saúde (LILACS) e da Medical Literature Analysis and Retrieval System Online (MEDLINE) e CINAHL (Figura 1).

Foram utilizados os descritores $<$ crianca $>$ e $<$ estomia $>$ e $<$ educacao $>$ de forma associada, para fazer uma busca integrada abrangendo o título, o resumo e as palavraschave. Este procedimento foi adotado a fim de proporcionar a viabilidade e abrangência deste estudo. Posteriormente, a leitura e a classificação dos resumos foram efetuadas por dois pesquisadores de maneira independente.

A amostra do estudo foi realizada a partir dos critérios de inclusão: estudos disponiveis em formato completo (full text) tais como: artigos de pesquisa, relatos de experiência ou revisão integrativa, relacionados a temática, nos idiomas português, inglês e espanhol, nos anos de 2013 até 2016. Como critério de exclusão foram incluídos: editoriais, cartas, artigos de opinião, comentários, notas prévias, publicações duplicadas, teses e dissertações, artigos completes que não estão dispostos na integra, livros, materiais 
publicações em outros idiomas que não português, inglês e espanhol.

As buscas dos dois examinadores foram comparadas, assim, nos casos em que o título e o resumo não foram esclarecedores, o artigo foi buscado na íntegra, sendo possíveis discordâncias resolvidas por consenso entre os pesquisadores participantes desta pesquisa.

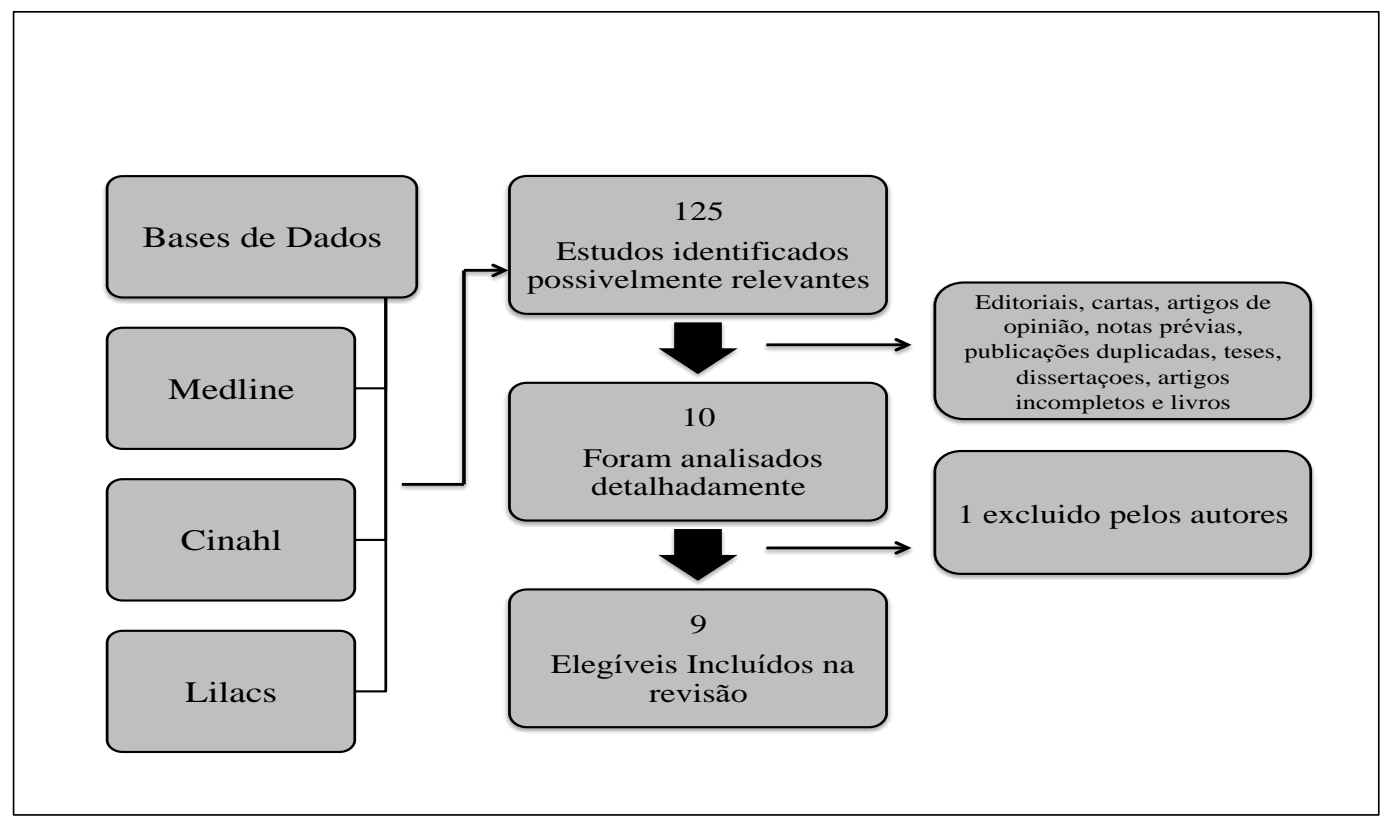

Figura 1: Fluxo da seleção dos estudos incluídos na revisão integrativa.

Fonte: Elaborado pelas autoras

Após a leitura dos trabalhos, as suas principais informações foram expressas em uma planilha, que contemplou: título, autores, revista, qualificação da produção intelectual da coordenação de aperfeiçoamento de pessoal de nível superior - QUALISCAPES, ano de publicação, objetivos, participantes, intervenção - procedimentos utilizados, local onde a pesquisa foi realizada (país), resultados obtidos, limites/dificuldades e conclusões/considerações finais. Em seguida, procedeu-se à análise dos dados.

\begin{tabular}{|c|c|c|c|c|c|}
\hline Autor & Titulo & Periódico & Qualis & Ano & País \\
\hline $\begin{array}{c}\text { Vilar, Andrade } \\
\text { \& Alves. }\end{array}$ & $\begin{array}{c}\text { Alta de crianças com estoma: } \\
\text { uma revisão integrativa da } \\
\text { literatura }\end{array}$ & $\begin{array}{c}\text { Revista de } \\
\text { Enfermagem } \\
\text { Referência }\end{array}$ & B2 & 2013 & Brasil \\
\hline $\begin{array}{c}\text { Rosado, Leite, } \\
\text { Silva, Dázio, } \\
\text { Terra \& Leite. }\end{array}$ & $\begin{array}{c}\text { Medidas para a assistência } \\
\text { domiliciar à criança com } \\
\text { estoma: revisao integrativa }\end{array}$ & $\begin{array}{c}\text { Rev enferm UFPE } \\
\text { on line. }\end{array}$ & B2 & 2013 & Brasil \\
\hline
\end{tabular}




\begin{tabular}{|c|c|c|c|c|c|}
\hline $\begin{array}{l}\text { Menezes, Góes, } \\
\text { Souza \& Maia. }\end{array}$ & $\begin{array}{l}\text { A autonomia da criança } \\
\text { estomizada: desafios para o } \\
\text { cuidado de enfermagem }\end{array}$ & $\begin{array}{l}\text { Rev enferm UFPE } \\
\text { on line }\end{array}$ & B2 & 2014 & Brasil \\
\hline $\begin{array}{l}\text { Zacarin, } \\
\text { Alvarenga, } \\
\text { Souza, Borges } \\
\text { \& Dupas. }\end{array}$ & $\begin{array}{l}\text { Vulnerabilidade da família de } \\
\text { crianças com estomia intestinal }\end{array}$ & Rev Eletr Enf & B1 & 2014 & Brasil \\
\hline $\begin{array}{l}\text { Rosado, Dázio, } \\
\text { Siepierski, } \\
\text { Filipini \& } \\
\text { Fava. }\end{array}$ & $\begin{array}{l}\text { O cuidado de Enfermagem e as } \\
\text { lacunas na assistência à criança } \\
\text { com Estomia }\end{array}$ & Revista Estima & B2 & 2015 & Brasil \\
\hline $\begin{array}{l}\text { Melo \& } \\
\text { Kamada. }\end{array}$ & $\begin{array}{l}\text { O papel da família no cuidado à } \\
\text { criança com estoma intestinal: } \\
\text { uma revisão narrativa }\end{array}$ & Revista Estima & B2 & 2015 & Brasil \\
\hline Chu \& Duong. & $\begin{array}{c}\text { Education of Parents When a } \\
\text { Child Born with an Imperforate } \\
\text { Anus; Does It Improve the } \\
\text { Health of the Child? }\end{array}$ & $\begin{array}{l}\text { Open Journal of } \\
\text { Pediatrics }\end{array}$ & $\begin{array}{l}\text { Impact } \\
\text { Factor } \\
0.44\end{array}$ & 2015 & Suécia \\
\hline $\begin{array}{l}\text { Dabas, } \\
\text { Sharma, Joshi } \\
\text { \& Agarwala. }\end{array}$ & $\begin{array}{l}\text { Video teaching program on } \\
\text { management of colostomy: } \\
\text { Evaluation of its impact on } \\
\text { caregivers. }\end{array}$ & $\begin{array}{l}\text { Journal of Indian } \\
\text { Association of } \\
\text { Pediatric } \\
\text { Surgeons. }\end{array}$ & $\begin{array}{l}\text { Impact } \\
\text { Factor } \\
0.59\end{array}$ & 2016 & Índia \\
\hline $\begin{array}{l}\text { Goudarzi, } \\
\text { Askari, Asgari } \\
\text { \& Mehran. }\end{array}$ & $\begin{array}{c}\text { The Effects of an } \\
\text { Empowerment Program on the } \\
\text { Stress Among Mothers of } \\
\text { Neonates Undergoing } \\
\text { Colostomy }\end{array}$ & $\begin{array}{l}\text { Journal of Critical } \\
\text { Care Nursing }\end{array}$ & A1 & 2016 & Iran \\
\hline
\end{tabular}

Quadro 1: Caracterização dos artigos revisados

Fonte: Elaborado pelas autoras

Todos os artigos que compõe a amostra foram submetidos a leitura minuciosa, realizada em duas etapas: na primeira realizou-se a síntese dos dados de identificação e caracterização da amostra e, na segunda, a análise de conteúdo dos artigos. Durante a fase de síntese foi possível perceber a diversidade nos métodos dos estudos, o que em muito acrescenta na reflexão acerca da abordagem do tema e as várias maneiras de se poder atingir a população estudada.

Optou-se pelo método de análise de conteúdo, que se explica por compreender técnicas de pesquisa que permitem, de forma sistemática, a descrição das mensagens e das atitudes atreladas ao contexto da enunciação, bem como as inferências sobre os dados coletados. A escolha também ocorreu pela necessidade de ultrapassar as incertezas consequentes das hipóteses e pressupostos, pela necessidade de enriquecimento da leitura por meio da compreensão das significações e pela necessidade de desvelar as relações que se estabelecem (CAVALCANTE; CALIXTO; PINHEIRO, 2014). Dessa forma na fase de análise de conteúdos originaram-se as quatro categorias com suas respectivas unidades de análise. 
Tabela 2: Produções científicas: níveis de evidência.

\begin{tabular}{|c|c|c|}
\hline Artigo & Método & Nível de Evidência \\
\hline $\begin{array}{l}\text { Alta de crianças com estoma: uma revisão integrativa } \\
\text { da literatura }\end{array}$ & Revisão integrativa & IV \\
\hline $\begin{array}{c}\text { Medidas para a assistência domiliciar à criança com } \\
\text { estoma: revisao integrativa }\end{array}$ & Revisão integrativa & IV \\
\hline $\begin{array}{c}\text { A autonomia da criança estomizada: desafios para o } \\
\text { cuidado de enfermagem }\end{array}$ & Qualitativa & VI \\
\hline $\begin{array}{l}\text { Vulnerabilidade da família de crianças com estomia } \\
\text { intestinal }\end{array}$ & Qualitativa & VI \\
\hline $\begin{array}{l}\text { O cuidado de Enfermagem e as lacunas na assistência } \\
\text { à criança com Estomia }\end{array}$ & Revisão integrativa & IV \\
\hline $\begin{array}{l}\text { O papel da família no cuidado à criança com estoma } \\
\text { intestinal: uma revisão narrativa }\end{array}$ & Revisão narrativa. & VI \\
\hline $\begin{array}{l}\text { Education of Parents When a Child Born with an } \\
\text { Imperforate Anus; Does It Improve the Health of the } \\
\text { Child? }\end{array}$ & Quanti e qualitativo & VI \\
\hline $\begin{array}{l}\text { Video teaching program on management of } \\
\text { colostomy: Evaluation of its impact on caregivers. }\end{array}$ & Estudo descritivo & IV \\
\hline $\begin{array}{c}\text { The Effects of an Empowerment Program on the } \\
\text { Stress Among Mothers of Neonates Undergoing } \\
\text { Colostomy }\end{array}$ & Ensaio clínico & II \\
\hline
\end{tabular}

Fonte: Elaborado pelas autoras

\section{Resultados}

A amostra da revisão integrativa foi composta por nove estudos (Quadro 1), sendo cinco primários e quatro secundários, com maior predominância das publicações no ano de 2015 (três estudos no ano). Em relação ao idioma, seis foram publicados em português e três em inglês, em periódicos, nacionais e internacionais, a saber: Revista Estima, Revista da UFPE, Revista eletrônica de enfermagem, Revista de enfermagem referência, Open jornal of Pediatrics, Online braz J nurs e Journal of Indian Association of Pediatric Surgeons, Journal of Critical Care Nursing. Quanto ao QUALIS/CAPES, as métricas dos periódicos foram: cinco B2, um (B1), um (A1) e dois em bases internacionais indicavam o fator de impacto de 0,44 e 0,59 respectivamente (Tabela 2). Percebe-se que a avaliação da qualidade e do desempenho das pesquisas utilizados nessa revisão estão em um padrão mediano.

Dos artigos analisados seis foram originados no Brasil e outros países como Suiça, Índia e Iran, com um trabalho respectivamente. Nos estudos primários foram encontrados 
dois estudos qualitativos com análise temática, um quanti-qualitativo, um estudo descritivo exploratório (quase-experimental) e um ensaio clínico, e os estudos secundários foram quatro revisões integrativas da literatura (Figura 2).

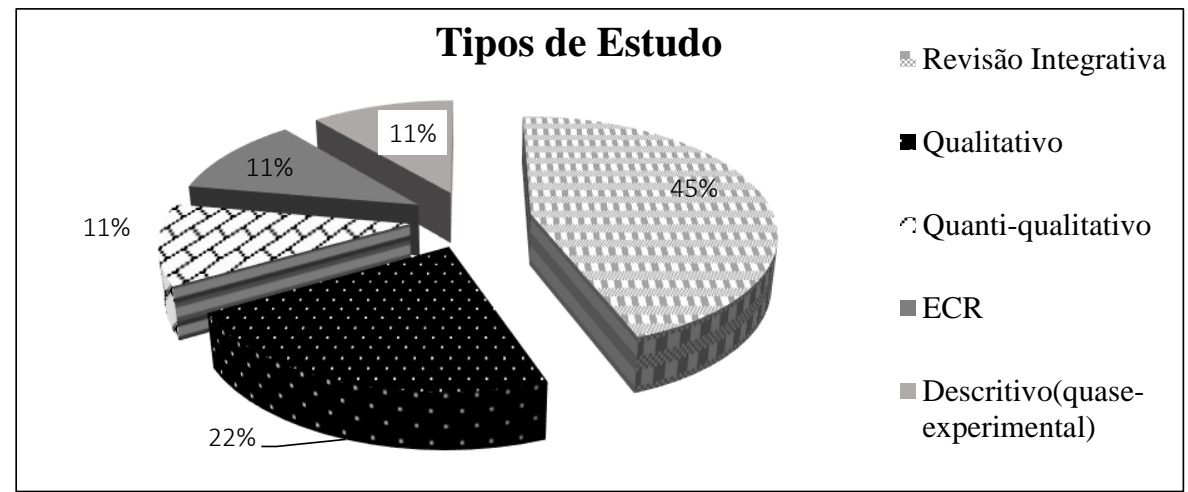

Figura 2: Tipo de estudos dos artigos analisados Fonte: Elaborado pelas autoras

A maioria (66,6\%) dos estudos foram escritos em português e 33,3\% em inglês, por enfermeiros, e em cada manuscrito haviam mestres e doutores em enfermagem vinculados a instituições de ensino superior, o que fornece credibilidade aos estudos e demonstra a preocupação dos profissionais com a assistência e a formação profissional.

Em relação ao nível de evidências dos periódicos teve variação de II, IV a VI, com predominância de estudo descritivo ou qualitativo; demonstrando a escuta qualificada, a sensibilidade do pesquisador, a subjetividade do indivíduo para trazer significados a prática assistencial, o que evidencia o quanto se tem a avançar na área de educação e saúde, tomando como referência os variados tipos de estudo.

Das investigações incluídas tiveram como temática central as mães/cuidadores de crianças estomizadas na execução dos cuidados diários com a estomia, pois tratava-se de crianças pequenas em pleno período de crescimento e desenvolvimento e portanto com inabilidade para autocuida-se. Por isso a menor idade foi prevalente na maioria dos estudos.

Os estudos evidenciaram a importância do trabalho do enfermeiro no cuidado de crianças estomizadas e seus familiares, ressaltando seu papel essencial no processo de reabilitação do estomizado. Acredita-se que esse foi um ponto forte da revisão, pois resgata sua identidade e o empodera na prática profissional por meio de medidas variadas que vão de uma simples conversa a complexos manejos assistenciais, porém que contribuem significativamente para a mudança de comportamento e atitudes das famílias frente as demandas cotidianas. 


\section{Discussão}

Para apresentar os resultados e discussões optamos por fazer análise de conteúdo, dessa forma surgiram quarto categorias temáticas: educação em saúde, atuação do enfermeiro, plano de cuidados e inserção social, discutidas a seguir.

\subsection{Educação em Saúde}

A preocupação com as informações, conhecimentos e orientações a serem repassadas aos pais/cuidadores de crianças estomizadas foi bem explorada nos manuscritos destacando a necessidade de capacitação técnica e humanização dos profissionais, com a finalidade da construção do conhecimento por meio da dialogicidade para amenizar o impacto da doença e o manejo indesejado com a estomia.

Faz-se necessário encorajar a família e não somente a mãe, que na maioria das vezes é a cuidadora principal, na participação dos cuidados e atividades da vida diária (VILAR; ANDRADE; ALVES, 2013; ROSADO et al., 2013; MELO; KAMADA, 2015; ROSADO et al., 2015).

A educação em saúde foi citada na maioria os artigos e traz como ponto forte do emponderamento e autonomia como resultados mais requeridos pelos familiares (VILAR; ANDRADE; ALVES, 2013; MENEZES et al., 2014; ZACARIN et al., 2014; ROSADO et al., 2015), pois os mesmos almejam se apropriarem de tal conhecimento para sentirem-se mais seguros e confiantes. Pesquisa recente sobre educação de pais de crianças estomizadas concluiu que a capacidade dos pais para superar os obstáculos depende em grande medida do seu conhecimento e confiança (CHU; DUONG, 2015). A educação em estomia para todos os níveis de prestadores de cuidados de saúde deve ser um processo sistemático, centrado no paciente e família, fornecido nos locais acadêmicos e locais de trabalho com nível de evidencia IV, considerado como bom no meio científico (RNAO, 2009).

Nesse sentido o plano de cuidado de enfermagem deve ser centrado na família, que tem a capacidade de potencializar forças e minimizar os problemas diante da condição de estomização. Nos últimos anos, os cuidados de saúde pediátricos têm sido centrados na família, pelo envolvimento e proximidade na participação dos cuidados, isso tem se demonstrado benéfico para a criança, pais e instituição (EKMAN et al., 2011). 
Há de existir e fortalecer o vínculo de profissionais cuidadores com os familiares, constituindo-se dessa forma um apoio familiar imprescindível. A educação em saúde se mostra como um potencializador de cuidados, pois as informações corretas apreendidas pelas pessoas contribuem por um estado de confiança e tranquilidade, além de serem disseminadas para outros de forma segura.

Em revisão integrativa recente acerca de educação em saúde, os outores concluiram que a educação é ferramenta essencial para desenvolvimento saudável de crianças com estomias, pois empoderando os pais/cuidadores e a própria criança a cerca dos motivos, funcionamento intestinal, complicações e cuidados a serem prestados, faz toda diferença no enfrentamento da condição (MONTEIRO et al., 2017).

\subsection{Atuação do Enfermeiro}

Quanto a atuação do enfermeiro foi citado a necessidade da elaboração de protocolos, criação de diretrizes e planejamento para ações educativas, gestão de competências e habilidades para a prática cotidiana até a alta, de forma sistematizada e individualizada, com vistas a adaptação iniciada ainda durante a internação, para que no momento da alta, a ida para o domicílio não seja um tormento (VILAR; ANDRADE; ALVES, 2013; MENEZES et al., 2014; ZACARIN et al., 2014; MELO; KAMADA, 2015).

Alguns estudos identificaram a falta de um planejamento sistemático e articulado, cada um faz de um jeito ou mesmo a ausência do plano de atuação, bem como, a falta de comunicação efetiva entre os profissionais e a família. Esse conjunto de falhas só tem a prejudicar a todos os envolvidos, e principalmente o paciente (ROSADO et al., 2015; VILAR; ANDRADE; ALVES, 2013).

Ensinar a criança maior é ajudar na construção de sua autonomia, e isso pode ser desenvolvido por meio de experiências do brincar livre, atividades de vida diárias típica para cada idade. Enfermeiras podem usar materiais didáticos como folhetos, cartilhas, desenhos, para educar pais e crianças submetidos a estomia (KALIA; WALIA; RAO, 2004). Eles podem informar aos pais/cuidadores sobre os problemas de seus filhos e assim aliviar o seu desespero e estresse (HOCKENBERRY; WILSON, 2012).

No âmbito do exercício profissional da enfermagem, o enfermeiro é considerado essencial no processo de reabilitação do estomizado, pois está presente desde o momento do diagnóstico, e indicação da confecção da estomia. Nesse ínterim, o enfermeiro orienta 
os estomizados a respeito dos cuidados com o estoma, alimentação, higienização, preparando-os para o cuidado de si e retorno às atividades de vida diária (TAYLOR; AZEVEDO-GILBERT; GABE, 2012).

Monteiro et al., (2017) afirmam que o enfermeiro é o elo de ligação entre os conhecimentos/informações e a práticas desses cuidados que devem ser ensinados desde o momento do conhecimento da necessidade da estomização até a alta hospitalar, por evidenciar melhora significativa nos cuidados domiciliares resultando em crianças mais saudáveis e pais mais confiantes.

Cabe aos profissionais pensar em estratégias de ações de saúde que transcendam o enfoque da doença, de modo a promover meios para auxiliar os usuários com estomia de eliminação a tomarem decisões, verbalizarem sentimentos e ajudá-los no enfrentamento das mudanças da sua imagem corporal em prol da sobrevivência (PEREIRA et al., 2012).

Percebe-se a importância do enfermeiro nas ações de saúde da população, o regaste de sua identidade como agente do cuidar e facilitador na reabilitação da criança com estomia. Recente estudo utilizando as intervenções de enfermagem no pósoperatório por meio de vídeo educativo para família de portadores de colostomia, foi considerada válida pelos participantes, mostrando-se esclarecedora e de aplicabilidade prática. $\mathrm{O}$ vídeo teve o potencial de ajudar em relação à aprendizagem dos cuidados com a estomia, aos aspectos emocionais e também na comunicação entre os familiares (STRAGLIOTTO et al., 2017).

\subsection{Plano de Cuidados}

As publicações informam que há um grande checklist de atividades para serem contempladas em prol da saúde do estomizado, entre elas está o plano de educação em saúde: sessões para discussão da função do sistema digestivo, via de defecação, a necessidade da estomia, complicações como inflamação, infecção, necrose, prolapso, retração do estoma, desidratação, risco de sangramento; sessões sobre cuidados com a criança: manejo do equipamento coletor, gerenciando o mal cheiro das fezes, monitoramento da saúde, alimentação, roupas, banho, viagens, acompanhamento e prognóstico, gerenciar e identificar emergências, realizar cuidados com a pele periestomia, limpeza e proteção do estoma; e sessões acerca de interpretação dos cuidados com a colostomia por parte dos pais/cuidadores e avaliação da sua capacidade de fornecer 
cuidados pelo enfermeiro (ROSADO et al., 2015; MENEZES et al., 2014; ZACARIN et al., 2014; ROSADO et al., 2013; MELO; KAMADA, 2015).

Apesar de ser apontadas as necessidades do cuidar no formato chekclist, não foi encontrado um instrumento físico propriamente dito para checagem dessas atividades. Percebe-se que cada profissional organiza seu serviço conforme seu entendimento e prioridade, podendo por vezes deixar de assistir um aspecto importante da saúde integral.

Todo esse cuidado deve ser repassado individualmente em forma de conversa (dialógo) e reforçado com uso de materiais educativos impressos, videos, brinquedo terapêntico (CHU; DUONG, 2015; DABAS et al., 2016; GOUDARZI et al., 2016).

O planejamento dos cuidados por meio de estratégias educativas deve ser sistemático e articulado para atender as necessidades de cada criança/família, além de propiciar possibilidades contínuas de discussão para reforçar a importância das melhores práticas (MONTEIRO et al., 2017).

As tecnologias cuidativas tem sido amplamente utilizadas para estreitar espaços entre o educar e o aprender, vistas como produtos sócio-operativos proveniente da vivência entre sujeitos, principalmente àqueles que possuem foco na mudança de comportamento para promoção da saúde e prevenção de agravos (ASSUNÇÃO et al., 2013).

É necessário permitir um tempo para que o familiar elabore e reflita acerca de tantas informações, por isso o ideal é que ocorram vários momentos de conversa para deixá-los à vontade a questionamentos, dúvidas, e assim expressarem seus sentimentos e dificuldades frente a nova condição do(a) filho(a). Cabe aos profissioanis de saúde apoiar momentos de enfrentamento negativo e positivo na condição de estomizado.

\subsection{Inserção social}

Recomenda-se evitar o confinamento domiciliar, incentivando a família a buscar apoio em rede social e/ou de amigos entre hospital/domicílio e rede de atenção básica (VILAR; ANDRADE; ALVES, 2013; MENEZES et al., 2014) para promover a inserção familiar e social da criança, a começar pela prática escolar visando a adaptação a realidade (ZACARIN et al., 2014; MELO; KAMADA, 2015; ROSADO et al., 2015; MENEZES et al., 2014). 
Compartilhar experiências com outras crianças de forma natural pode transcender a estigmatização que surge em torno da pessoa com estoma, incentivando a socialização e a melhora na qualidade de vida.

Em três estudos da amostra, os autores utilizaram como estratégias de educação em saúde: um programa de educação estruturado, um programa educacional utilizando vídeo com informações inerentes a estomização, e um programa de capacitação educacional para pais, utilizando grupo de intervenção e o grupo controle (CHU; DUONG, 2015; DABAS et al., 2016; GOUDARZI et al., 2016).

Nesses estudos a conclusão que se chegou é que a educação dos pais de crianças estomizadas melhora consideravelmente a saúde da criança, as emoções das mães mudaram e se tornaram positivas, quando conheciam o passo a passo da condição do estomizado, ainda mais se forem apoiados pela equipe de saúde, pois sentiam-se mais confiantes para cuidar do(a) filho(a) (CHU; DUONG, 2015).

A utilização de material didático audiovisual no ensino de pais/cuidadores de crianças estomizadas tem sido para melhorar seus conhecimentos e habilidades, ocorrendo um aumento significativo nas pontuações de conhecimento imediatamente após o uso, bem como num período de duas semanas após o vídeo (DABAS et al., 2016).

O programa de capacitação educacional para pais/cuidadores revelou ser eficaz em alívio do estresse das mães por fornecer informações de saúde de seus filhos, além de capacitá-las para melhor prestação de cuidados, dessa forma a implementação de programas como esse torna-se essencial nos serviços de saúde (GOUDARZI et al., 2016).

Observa-se a importância da socialização da criança com estomia no meio familiar, escolar e social e que a estrategia de educação em saúde para pais, familares, professores, amigos e pessoas interessadas é o diferencial para um bom enfrentamento de estomias pediátricas, para comprovar que a criança pode ter uma vida normal sem preconceitos e/ou estigmas.

As contribuições dessa pesquisa ancoram-se no conhecimento sobre as boas práticas do enfermeiro e traz visibilidade para os benefícios da educação em saúde. A limitação do estudo foi ter encontrado poucos estudos primários que apresentassem estratégias de educacão em saúde paras as crianças, pois a maioria dos estudos foram voltados aos pais e cuidadores. 


\section{Conclusão}

As publicações analisadas revelaram que a educação em saúde é ferramenta essencial para desenvolvimento saudável de crianças com estomias intestinais. Empoderar aos pais/cuidadores e a criança a cerca dos motivos, funcionamento intestinal, complicações e cuidados a serem prestados faz toda diferença no enfrentamento de quem precisa passar por essa condição.

O enfermeiro é o elo de ligação entre os conhecimentos/informações e a práticas desses cuidados que devem ser ensinados desde o momento do conhecimento da necessidade da estomização até a alta hospitalar, por evidenciar melhora significativa nos cuidados domiciliares resultando em crianças mais saudáveis e pais mais confiantes.

O planejamento dos cuidados por meio de estratégias educativas deve ser sistemático e articulado para atender as necessidades de cada criança/família, além de propiciar possibilidades contínuas de discussão para reforçar a importância das melhores práticas.

\section{Referências}

ASSUNÇÃO, A. P. F. et al. Práticas e tecnologias educacionais no cotidiano de enfermeiras da estratégia saúde da família. Rev enferm UFPE on line., Recife, v.7, n.11, p. 6329-35, nov. 2013.

BEZERRA, P. D. et al. Perfil sociodemográfico e clínico de crianças com estomia atendidas em um serviço de referência. Estima, Belém, v.15, n.4, p. 214-221, out./dez. 2017.

CAVALCANTE, R. B.; CALIXTO, P.; PINHEIRO, M. M. K. Análise de contéudo. Considerações gerais, relações com a Pergunta de pesquisa, possibilidades e limitações do método. Inf. \& Soc., João Pessoa, v.24, n.1, p.13-18, jan./abr. 2014.

CHU, T. H.; DUONG, T. H. Education of Parents When a Child Born with an Imperforate Anus; Does It Improve the Health of the Child? Open Journal of Pediatrics, v.5, p.90-98, mar. 2015.

DABAS. H. et al. Video teaching program on management of colostomy: Evaluation of its impact on caregivers. Journal of Indian Association of Pediatric Surgeons, New Delhi, India, v. 2 , n. 21, p.54-56, feb. 2016.

EKMAN, I. et al. Person Centered Care-Ready for Prime Time. European Journal of Cardiovascular Nursing, Suécia v.10, n.4. p .248-251, dec.2011.

FONSECA, E.; MARCON, S. Support network to families of low birth weight babies after hospital discharge: a qualitative study. Online braz j nurs [internet], Niterói, Rio de Janeiro v.8, n.2, p. 1-8, may./aug. 2009. 
GOUDARZI, Z. et al. The Effects of an Empowerment Program on the Stress Among Mothers of Neonates Undergoing Colostomy. Crit Care Nurs J, [online], v.9, n.1, p. 5098, feb. 2016.

HOCKENBERRY, M.J.; WILSON, D. Wong's Essentials of Pediatr Nursing. 10. ed. Pennsylvania, United States: Elsevier Health Sciences. 2012.

KALIA, R.; WALIA, I.; RAO, K.L. Developing of educational aids for the parents of children having colostomy. J Indian Assoc Pediatr Surg, Toronto, v.9, p.15-9, 2004.

KAYO, C.M.M. et al. Cuidando de Crianças com Estomia. In: SANTOS, V. L. C. G.; CESARETTI, I. U. R. Assistência em Estomaterapia: Cuidando de Pessoas com Estomia. 2. ed. São Paulo: Atheneu, 2015. p. 205-242.

MATSUBARA, M.G.S. et al. Feridas e Estomas em Oncologia: uma Abordagem Interdisciplinar. 1. ed. São Paulo: Lemar, 2012.

MELO, C. M.; KAMADA, I. O papel da família no cuidado à criança com estoma intestinal: uma revisão narrativa. Revista Estima, São Paulo. v.13, n.3, p.121-126, jul./set. 2015.

MENDES, K. D. S.; SILVEIRA, R. C. C. P.; GALVÃO, C. M. Revisão integrativa: método de pesquisa para a incorporação de evidências na saúde e na enfermagem. Texto \& Contexto Enfermagem, Florianópolis v.17, n.4, p.758-764, out./dez. 2008.

MENEZES, H. F.; et al. A autonomia da criança estomizada: desafios para o cuidado de enfermagem. Rev enferm UFPE on line, Recife, v.8, n.3, p.632-40, mar. 2014.

MONTEIRO, S. N. C. et al. Perfil de crianças e adolescentes estomizados atendidos de um hospital público do Distrito Federal. Revista Estima, São Paulo. v.12, n.3, p.23-32, abr./jun. 2014.

MONTEIRO, S. N. C. et al. Atuação do enfermeiro na educação em saúde de crianças com estomias intestinais: revisão integrativa. In: CONGRESSO IBEROAMERICANO DE PESQUISA QUALITATIVA, 6, 2017, Salamanca. Anais... Salamanca: Universidade Lusófona do Porto, 2017. p 345-354.

PEREIRA, A.P.S. et al. Associations among sociodemographic and clinical factors and the quality of life of ostomized patients. Rev. Latino-Am. Enfermagem, Ribeirão Preto, v.20, n. 1, p.93-100, jan./feb. 2012.

ROSADO, S.R. et al. Medidas para a assistencia domiliciar à criança com estoma: revisão integrativa. Rev enferm UFPE on line, Recife, v.7, n. esp., p.7175-80, dez. 2013.

ROSADO, S.R. et al. O cuidado de Enfermagem e as lacunas na assistência à criança com Estomia. Revista Estima, São Paulo, v.13, n.2, p.83-87, abr./jun. 2015.

SANTOS, V.L.C.G.; CESARETTI, I.U.R. Assistência em Estomaterapia: Cuidando de Pessoas com Estomia. 2. ed. São Paulo: Atheneu, 2015.

STRAGLIOTTO, D.O. et al. Implementação e avaliação de um vídeo educativo para famílias e pessoas com colostomia. Revista Estima, São Paulo, v.15 n.4, p. 191-199, out./dez. 2017.

TAYLOR, C.; AZEVEDO-GILBERT, R.L.; GABE, S. Rehabilitation needs following stoma formation: apatient survey. Br J Community Nurs, London, v.17, n.3, p.102-107, aug. 2012. 
TEIXEIRA. E.; MEDEIROS. H. P.; NASCIMENTO, M. H. M. Referenciais metodológicos para validação de tecnologias cuidativo-educacionais. In: NIETSCHE, E. A.; TEIXEIRA, E.; MEDEIROS, H. P. Tecnologias cuidativo-educacionais: uma possibilidade para o mpoderamentodo (a) enfermeiro(a)? 1. ed. Porto Alegre: Moriá, 2014. p.113-127.

VILAR, A.M.A.; ANDRADE, M.; ALVES, M.R.S. Alta de crianças com estoma: uma revisão integrativa da literatura. Revista de Enfermagem Referência, Coimbra, v.3, n.10, p.145-152, jul. 2013.

ZACARIN, C.F.L. et al. Vulnerabilidade da família de crianças com estomia intestinal. Rev. Eletr. Enf. [Internet], Goiás. v. 16, n.2, p. 426-33, abr./jun. 2014.

Recebido em: 24 de março de 2018.

Aceito em: 05 de abril de 2018 . 